Takehiro Izumo', Yuriko Terada ${ }^{2}$, Minoru Inomata', Naoyuki Kuse', Nobuyasu Awano', Mari Tone', Tatsunori Jo', Hanako Yoshimura', Atsuko Moriya', Yoshiaki Furuhata ${ }^{2}$

'Department of Respiratory Medicine, Japanese Red Cross Medical Center, Tokyo, Japan

${ }^{2}$ Department of Thoracic Surgery, Japanese Red Cross Medical Center, Tokyo, Japan

\title{
Impact of preoperative pathological confirmation on surgical and postoperative outcomes of lung resection for early stage lung cancer
}

\begin{abstract}
Introduction: The frequency of detection of peripheral pulmonary lesion (PPL) in suspected early lung cancer has been increasing, and whether preoperative pathological diagnosis (PPD) for small PPLs should always be established before their surgical resection can become a worrisome problem for physicians. The aim of the study was to clarify the impact of obtaining PPD on surgical and postoperative outcomes of lung resection for early stage lung cancer.

Material and methods: This was a retrospective review of cases that underwent surgical resection for known or suspected primary lung cancer presenting pathological stage 0 or I, enrolled from June 2006 to May 2016. The patients divided into two groups according to PPD group $(n=57)$ and non-PPD group $(n=157)$ were compared. The procedure, node dissection, operation time, amount of bleeding, postoperative complications, postoperative length of stay, and postoperative recurrences were analyzed.

Results: Among the 214 patients, no significant differences in operation time $(248.5 \pm 88.6$ versus $257.6 \pm 89.0$, min, mean $\pm \mathrm{SD}, \mathrm{p}=0.328)$, amount of bleeding $(195.3 \pm 176.5 \mathrm{vs} 188.1 \pm 236.1, \mathrm{ml}, \mathrm{p}=0.460)$, postoperative complication $(5.2 \%$ vs $4.5 \%, p=0.728)$, postoperative length of stay $(10.6 \pm 6.3$ vs $10.4 \pm 5.3$, days, $p=0.827)$, or postoperative recurrences $(21.0 \%$ vs $17.2 \%, \mathrm{p}=0.550$ ) were seen between PPD and non-PPD groups.

Conclusion: Therefore, PPD had less impact on surgical and postoperative outcomes of pathological stage 0 or I lung cancer; direct surgical resection without non-surgical biopsy would be acceptable with careful selection of cases.
\end{abstract}

Key words: lung cancer, transbronchial biopsy, CT-guided needle biopsy, peripheral pulmonary lesion, video-assisted thoracic surgery

Adv Respir Med. 2019; 87: 203-208

\section{Introduction}

Lung cancer is one of the most important causes of cancer-related deaths over the world [1-3]. Since the widespread using of low-dose computed tomography (CT) screening promised to reduce lung cancer mortality, the detection and diagnosis rate of peripheral pulmonary lesions (PPLs) has increased [4]. PPLs have a high incidence of lung cancer at an early stage [4]. To improve the survival outcomes of lung cancer, prompt surgical resection that is a preferred treatment for localized lung cancer is important. However, it involves the risk of unnecessary resections of benign lesions when preoperative definitive diagnosis is not established. The unnecessary surgical resection rates for benign diseases are reported to be from $9 \%$ to $40 \%[5,6]$. Therefore, preoperative pathological confirmations of PPLs by non-surgical biopsy, such as CT-guided needle biopsy and bronchoscopy, are important to avoid unnecessary surgical resections. In the recent study, the preoperative pathological diagnosis (PPD) by non-surgical biopsy have resulted in a low rate of benign resection [7].

Ideally, PPD for PPLs, which can represent benign diseases as well as localized lung cancer, should always be established by non-surgical mo-

Address for correspondence: Takehiro Izumo, Department of Respiratory Medicine, Japanese Red Cross Medical Center, 4-1-22 Hiroo Shibuya-ku, Tokyo, 150-8935 Japan; e-mail: izumo_takehiro@med.jrc.or.jp

DOI: 10.5603/ARM.a2019.0034

Received: 01.05.2019

Copyright (C) 2019 PTChP

ISSN 2451-4934 
dalities before their surgical resections. Although ideal, in the clinical settings surgical resections without PPD are often performed considering the high death rate of unresected lung cancer; besides there is a question of uncertainty, difficulty and risk of complications of non-surgical biopsy [8]. Furthermore, a recent study reported that following the recommendation of CT screening protocol has decreased the rate of benign resection by less than $2 \%$ and has not missed lung cancer cases [5]. Therefore, there is a controversy on the real benefit of non-surgical biopsy for PPLs in suspected lung cancer on a routine basis.

The aim of the study was to clarify the impact of obtaining PPD on surgical and postoperative outcomes of lung resection of early stage lung cancer, which would provide both patients and doctors with useful information about whether to decide or not to perform non-surgical biopsy routinely.

\section{Material and methods}

\section{Patients}

Consecutive patients who underwent surgical resection for known or suspected first primary lung cancer presenting pathological stage 0 or I at Japanese Red Cross Medical Center, between June 2006 and May 2016 were enrolled. Patients with multiple pulmonary lesions, those with history of cancer treated within past 5 years, those who were postoperatively diagnosed as pathological stage II-IV lung cancer, and those finally diagnosed with benign disease were excluded from the study. High-resolution computed tomography (HRCT) or 18-fluoro-deoxyglucose positron emission tomography (FDG-PET) were undertaken before surgery for all patients staged according to the TNM Classification of Malignant Tumors, seventh edition. CT-guided needle biopsy or bronchoscopy were performed for PPD of PPLs according to the physician's discretion.

All operations were video-assisted thoracic surgery (VATS). Sublobar resection (segmentectomy or wedge resection) was allowed in cases assessed intraoperatively as non-invasive adenocarcinoma and cases with no lymph node metastasis regarded as unsuitable for lobectomy considering factors such as age, pulmonary function, and general condition. Data on patient characteristics, chest CT and FDG-PET findings of PPLs, results of preoperative diagnostic examination performed in cases (diagnosed or undiagnosed), and patients' outcomes were retrospectively analyzed. This retrospective study was approved by the Institutional Review Board of Japanese Red Cross Medical Center (No. 912, UMIN000033593).

\section{Assessment}

To evaluate the impact of obtaining PPD on surgery, postoperative records in the patients with PPD (PPD group) and in the patients without PPD (non-PPD group) were compared. The surgical records were analyzed as follows: procedure (lobectomy or sublobar resection), node dissection (no lymphadenectomy, hilar lymphadenectomy, hilar and mediastinal lymphadenectomy), operation time, amount of bleeding, postoperative complications ( $\geq$ grade 3 ), postoperative length of stay, and postoperative recurrences. The Fischer exact test for categorical data and Mann-Whitney $\mathrm{U}$ test for numeric data were used to evaluate the significance of difference in surgical and preoperative records observed in the PPD and non-PPD groups. Descriptive statistics presented in the study are mean \pm standard deviation, frequency, and percentage. All reported $p$ values were two-sided, and a $p$ value $<0.05$ was considered statistically significant. All statistical analyses were performed using EZR (Saitama Medical Center, Jichi Medical University, Saitama Japan), which is a graphical user interface for R (The R Foundation for Statistical Computing, Vienna, Austria) [9].

\section{Results}

There were 214 patients who underwent surgical resection for known or suspected lung cancer presenting pathological stage 0 or I during the study period; 57 patients with PPD and 157 patients without PPD. All Stage 0 (TisN0M0) cases presented with a pure ground glass nodule (GGN). 59 subjects (23 patients in the PPD group and 36 patients in the non-PPD group) underwent $\mathrm{PET} / \mathrm{CT}$ in the study. Intraoperative diagnosis was performed in all non-PPD patients. The study population had a mean age of 66.9 \pm 9.8 years (mean \pm SD) and mostly consisted of male patients. The mean lesion size was $21.7 \pm 9.2 \mathrm{~mm}$ (mean $\pm \mathrm{SD}$ ). A summary of patient characteristics is shown in Table 1. Of the 214 patients, examinations for PPD were performed in 116 subjects (111 patients by bronchoscopy and five patients by CT-guided needle biopsy), and 57 individuals were successfully diagnosed (52 patients by bronchoscopy and five persons by CT-guided needle biopsy) (Table 2). With respect to complications, no severe complications were observed in the present study. 
Table 1. Baseline characteristics of 214 lung cancer patients with and without preoperative tissue diagnosis

\begin{tabular}{|c|c|c|c|c|c|}
\hline Variables & & $\begin{array}{c}\text { All patients } \\
\text { (n=214) }\end{array}$ & $\begin{array}{l}\text { PPD group } \\
(\mathrm{n}=57)\end{array}$ & $\begin{array}{l}\text { Non-PPD group } \\
\quad(\mathbf{n}=157)\end{array}$ & $P$ value \\
\hline Age (years) & & $66.9 \pm 9.8$ & $67.0 \pm 9.2$ & $66.5 \pm 10.0$ & 0.79 \\
\hline \multirow[t]{2}{*}{ Gender, no. $(\%)$} & Male & $140(65.4)$ & $41(71.9)$ & $99(63.1)$ & 0.26 \\
\hline & Female & $74(34.6)$ & $16(28.1)$ & $58(36.9)$ & \\
\hline \multirow[t]{2}{*}{ Smoking history } & Yes & $141(65.9)$ & $38(66.7)$ & $103(65.6)$ & 1.0 \\
\hline & No & $73(34.1)$ & $19(33.3)$ & $54(34.4)$ & \\
\hline Lesion size, mm & & $21.7 \pm 9.2$ & $25.2 \pm 8.9$ & $20.4 \pm 9.0$ & 0.01 \\
\hline \multirow[t]{3}{*}{ Pathological stage } & 0 & $12(5.6)$ & $2(3.5)$ & $10(6.4)$ & 0.10 \\
\hline & la & $122(57.0)$ & $27(47.4)$ & $95(60.5)$ & \\
\hline & $\mathrm{lb}$ & $80(37.4)$ & $28(49.1)$ & $52(33.1)$ & \\
\hline \multirow[t]{3}{*}{ Histologic subtype } & Adenocarcinoma & $153(71.5)$ & $41(71.9)$ & $112(71.3)$ & 0.49 \\
\hline & Squamous cell carcinoma & $45(21.0)$ & $10(17.5)$ & $35(22.3)$ & \\
\hline & Others & $16(7.5)$ & $6(10.6)$ & $10(6.4)$ & \\
\hline
\end{tabular}

Variables are presented as the mean $\pm \mathrm{SD}$ or $\mathrm{n}(\%) . P$ value is compared between PPD group and Non-PPD group

Table 2. Preoperative diagnostic modality for lesions suspected lung cancer

\begin{tabular}{lccc}
\hline Procedure & No. of examination & No. of cases obtained PPD & Percentage (\%) \\
\hline Bronchoscopy & 111 & 52 & 46.8 \\
CT-guided biopsy & 5 & 5 & 100 \\
\hline
\end{tabular}

CT — computed tomography; PPD — preoperative pathological diagnosis

Table 3. Comparison of surgical and postoperative outcomes between patients with PPD and those without PPD

\begin{tabular}{|c|c|c|c|c|}
\hline Variables & & $\begin{array}{l}\text { PPD group } \\
(\mathrm{n}=57)\end{array}$ & $\begin{array}{l}\text { Non-PPD group } \\
\quad(\mathrm{n}=157)\end{array}$ & $P$ value \\
\hline \multirow[t]{2}{*}{ Procedure } & Lobectomy & $52(91.2 \%)$ & $121(77.1 \%)$ & 0.02 \\
\hline & Sublobar resection & $5(8.8 \%)$ & $36(22.9 \%)$ & \\
\hline Node dissection & ND0/ND1/ND2 & $1(1.8 \%) / 2(3.6 \%) / 54(94.6 \%)$ & $13(8.2 \%) / 15$ (9.6\%) / 129 (82.2\%) & 0.07 \\
\hline Operation time, $\min$ & & $248.5 \pm 88.6$ & $257.6 \pm 89.0$ & 0.33 \\
\hline Amount of bleeding, $\mathrm{ml}$ & & $195.3 \pm 176.5$ & $188.1 \pm 236.1$ & 0.46 \\
\hline $\begin{array}{l}\text { Postoperative complication } \\
\text { ( } \geq \text { grade } 3 \text { ) }\end{array}$ & & $3(5.2 \%)$ & $7(4.5 \%)$ & 0.73 \\
\hline $\begin{array}{l}\text { Postoperative length of stay, } \\
\text { days }\end{array}$ & & $10.6 \pm 6.3$ & $10.4 \pm 5.3$ & 0.83 \\
\hline Postoperative recurrences & & $12(21.0 \%)$ & $27(17.2 \%)$ & 0.55 \\
\hline
\end{tabular}

Variables are presented as the mean \pm SD or $n(\%)$

NDO — no lymphadenectomy; ND1 — hilar lymphadenectomy; ND2 — hilar and mediastinal lymphadenectomy; PPD — preoperative pathological diagnosis

Among the 214 patients, no significant differences in operation time, amount of bleeding, postoperative complication, postoperative length of stay, or postoperative recurrences were seen between the PPD and non-PPD groups $(P=0.33$,
$P=0.46, P=0.73, P=0.83$, and $P=0.55$, respectively), but the rate of lobectomy was significantly higher in the PPD group (91.2\%) than in the non-PPD group $(77,1 \% P=0.02)$ (Table 3 ). In the PPD group, 8 patients had local recurren- 
ce and 4 patients had distant recurrence. In the non-PPD group, 18 subjects had local recurrence and 9 patients had distant recurrence. During this present study period, 58 patients underwent surgical resection for stage II-IV (30 patients for stage II, 27 patients for stage III and 1 person for stage IV) in the non-PPD way.

\section{Discussion}

To our knowledge, this is the first report on the impact of preoperative pathological confirmation on surgical and postoperative outcomes of lung resection of early stage lung cancer. The frequency of detection of PPL in suspected early lung cancer has been increasing, and whether PPD for small PPLs should always be established before their surgical resection can become a worrisome problem for physicians. Previous studies have reported the high resection rates (21-34\%) of benign lesions when preoperative pathological malignancy was not confirmed $[5,10]$. In the clinical situation, diagnosis of small benign lesions at peripheral pulmonary lesions are sometimes very difficult. However, in the present study, no significant differences in operation time, amount of bleeding, postoperative complication, postoperative length of stay, or postoperative recurrences were seen between the PPD and non-PPD groups. This result suggests that obtaining PPD has less impact on surgical and postoperative outcomes of pathological stage 0 or I lung cancer except for decreasing benign resections. In the current study, 27 patients who underwent surgical resection for suspected lung cancer in the non-PPD group had benign lesions. In clinical practice, it is very difficult to diagnose "benign" with small specimens such as bronchoscopy or CT-guided needle biopsy. The cause is that only a part of the lesion can be sampled by those procedures. For these reasons, the cases finally diagnosed as malignant were analyzed in the current study.

When there is a suspicion that the PPL is malignant, either CT-guided needle biopsy or bronchoscopy is often performed as non-surgical biopsy for PPD. The diagnostic yield of CT-guided needle biopsy for PPLs has been reported to be more than 90\% [11]. However, the procedure is often complicated by pneumothorax (29.8\%) and occasionally more serious complications such as tension pneumothorax $(0.10 \%)$, tumor seeding $(0.06 \%)$, or air embolism $(0.06 \%)$ [12]. In contrast, bronchoscopy is a safe diagnostic method for peripheral pulmonary lesions. Pneumothorax reported as the most frequent complication asso- ciated with bronchoscopy occurs with an incidence of around 1.0\% [13]. Moreover, the diagnostic value of bronchoscopy has recently improved as a consequence of using new guiding techniques such as endobronchial ultrasound (R-EBUS), guide sheath, virtual bronchoscopy, and virtual fluoroscopy, so that bronchoscopy seems to be a feasible diagnostic modality [14-16]. However, previous studies have reported the decreasing diagnostic yield of the bronchoscopy for the lesions measuring less than $20 \mathrm{~mm}$, those with negative bronchus sign on CT and those with GGNs [17].

Unfortunately, clinical stage 0 or I lung cancer often includes those kinds of lesions. Therefore, physicians often puzzle over the problem which preoperative diagnostic modality would be the most suitable for lesions in suspected pathological stage 0 or I lung cancer. On the other hand, surgical biopsy is a well-established and precise diagnostic modality for diagnosing PPLs. Importantly, the operation can consecutively be extended to include curative resection followed by surgical biopsy and confirmation of malignancy of lesions. If the lesions had highly suspicious characteristics of pathological stage 0 or I lung cancer on CT or FDG-PET, are there any advantages of preoperative pathological confirmation over the surgical and postoperative outcomes?

In the present study, preoperative pathological confirmation had no effect on the surgical or postoperative outcomes. According to those results, in the PPLs of highly suspected lung cancer with pathological stage 0 or I, surgical resection without $\mathrm{PPD}$ seems to be one of the feasible therapeutic strategies to avoid unnecessary complications of examination and delay to surgery.

A core needle biopsy or wedge lung resection is commonly performed as surgical biopsy. Since the advent of VATS, the patient with undiagnosed PPL could have undertaken minimally invasive surgical biopsy using VATS approach compared to conventional open thoracotomy. In most of the cases with surgical biopsy using VATS approach, extending operation, including curative resection is decided after confirmation of frozen section results. In other words, the operation including curative resection of undiagnosed lesion requires waiting time for intraoperative frozen section analysis. Therefore, there were concerns over the prolonged operation time. In the present study, no significant differences in operation time were found between the PPD and non-PPD groups. With regard to surgical and postoperative outcomes, no significant differences were found 
between the PPD and non-PPD groups. In the clinical setting, peeling off the area of pleural adhesions and preliminary dissection of the hilar structures, including lymph node to get ready for lobectomy can be started while waiting for the frozen section analysis, reducing waste of time. Moreover, previous studies have evaluated the risk factors associated with prolonged operation and postoperative complications, including pleural adhesion and incomplete fissure [18-20]. On the basis of our results and previous reports, we presume the absence of preoperative pathological diagnosis does not affect the surgical and postoperative outcomes of VATS of pathological stage 0 or I lung cancer except for decreasing the rate of unnecessary resection.

Today sublobar resection has been allowed for appropriate patients with the size of lesion $\leq 20 \mathrm{~mm}$, GGN with its consolidation tumor ratio $\leq 50 \%$, GGN with adenocarcinoma-in-situ, and lesion demonstrating a radiologic doubling time of $\geq 400$ days, and they often exhibit pathological stage 0 or I non-small cell lung cancer [21]. However, most physicians encounter clinical difficulties identifying those lesions by non-surgical diagnosis due to their small size, absence of bronchus which reaches into the lesion, or their minimal changes in histology. In those lesions, if lung cancer is highly suspected, sublobar resection using VATS approach can safely provide both pathological diagnosis and curative treatment with minimal invasiveness, saving costs and avoiding complications of non-surgical biopsy.

If suspicion of malignancy is not so strong, the probability of unnecessary resection of undiagnosed PPLs can be decreased by analysis of radiologic characteristics and careful CT follow-up examination [22, 23]. Additionally, in the real world, sublobar resection has also been allowed in non-small cell lung cancer cases with no lymph node metastasis considered unsuitable for lobectomy taking into account patient conditions (e.g., advanced age, coexisting comorbidities of chronic heart failure, and limited pulmonary function due to chronic obstructive pulmonary disease or interstitial lung disease). Considering the patient delicate conditions and risk of complications associated with non-surgical biopsy, there has been pros and cons whether non-surgical biopsy should be performed in cases with lesions highly suggesting early-stage non-small cell lung cancer.

The present study has several limitations. First, it was retrospective, nonrandomized, and conducted at a single medical center. Second, the study did not evaluate how often preoperative examination decrease the benign resection rates of undiagnosed PPLs, which are one of the main purpose of bronchoscopy and CT-guided biopsy. Additionally, obtaining PPD is ideal in the lesions which have suspicion of primary lung cancer as well as metastases derived from other organs, which may influence selection of surgery treatment. Third, the main preoperative diagnostic modality was bronchoscopy, which is reflective of the mode of operation in our institution. This may not be generalizable to other hospitals that mainly perform CT-guided biopsy for PPD. Prospective, randomized multicenter studies are needed for more accurate analysis.

\section{Conclusions}

The results of the present study show that obtaining PPD had less impact on surgical and postoperative outcomes of pathological stage 0 or I lung cancer, which suggests that direct surgical resection without an attempt to perform non-surgical biopsy would be acceptable with careful selection of cases.

\section{Author's contribution}

TI and YT designed the study, collected and analyzed the data, and wrote the paper. MI, NK, NA, MT, TJ, HY, AM and YF designed the study and collected the data.

\section{Conflict of interest}

All authors have no conflicts of interest to disclose.

\section{References:}

1. Gatta G, Zigon G, Capocaccia R, et al. EUROCARE Working Group. Survival of European children and young adults with cancer diagnosed 1995-2002. Eur J Cancer. 2009; 45(6): 9921005, doi: 10.1016/j.ejca.2008.11.042, indexed in Pubmed: 19231160.

2. Matsuda T, Marugame T, Kamo KI, et al. Japan Cancer Surveillance Research Group. Cancer incidence and incidence rates in Japan in 2006: based on data from 15 population-based cancer registries in the monitoring of cancer incidence in Japan (MCIJ) project. Jpn J Clin Oncol. 2012; 42(2): 139-147, doi: 10.1093/jjco/hyr184, indexed in Pubmed: 22172347.

3. Siegel R, Naishadham D, Jemal A. Cancer statistics, 2013. CA Cancer J Clin. 2013; 63(1): 11-30, doi: 10.3322/caac.21166, indexed in Pubmed: 23335087.

4. Kaneko M, Eguchi K, Ohmatsu H, et al. Peripheral lung cancer: screening and detection with low-dose spiral CT versus radiography. Radiology. 1996; 201(3): 798-802, doi: 10.1148/ radiology.201.3.8939234, indexed in Pubmed: 8939234.

5. Flores R, Bauer T, Aye R, et al. I-ELCAP Investigators. Balancing curability and unnecessary surgery in the context of computed tomography screening for lung cancer. J Thorac Cardiovasc Surg. 2014; 147(5): 1619-1626, doi: 10.1016/j. jtcvs.2013.11.001, indexed in Pubmed: 24332102. 
6. Carillo GA, Vázquez JE, Villar AF. Prevalence of benign pulmonary lesions excised for suspicion of malignancy: could it reflect a quality management index of indeterminate lung lesions? Korean J Thorac Cardiovasc Surg. 2014; 47(5): 458464, doi: 10.5090/kjtcs.2014.47.5.458, indexed in Pubmed: 25346901.

7. Yang W, Sun W, Li Q, et al. Diagnostic accuracy of ct-guided transthoracic needle biopsy for solitary pulmonary nodules. PLoS One. 2015; 10(6): e0131373, doi: 10.1371/journal. pone.0131373, indexed in Pubmed: 26110775.

8. Gould MK, Donington J, Lynch WR, et al. Evaluation of individuals with pulmonary nodules: when is it lung cancer? Diagnosis and management of lung cancer, 3rd ed: American College of Chest Physicians evidence-based clinical practice guidelines. Chest. 2013; 143(5 Suppl): e93S-e93e120S, doi: 10.1378/chest.12-2351, indexed in Pubmed: 23649456.

9. Kanda Y. Investigation of the freely available easy-to-use software "EZR" for medical statistics. Bone Marrow Transplant. 2013; 48(3): 452-458, doi: 10.1038/bmt.2012.244, indexed in Pubmed: 23208313.

10. Wilson DO, Weissfeld JL, Fuhrman CR, et al. The pittsburgh lung screening study (pluss): outcomes within 3 years of a firs computed tomography scan. Am J Respir Crit Care Med. 2008; 178(9): 956-961, doi: 10.1164/rccm.200802-3360C, indexed in Pubmed: 18635890.

11. DiBardino DM, Yarmus LB, Semaan RW. Transthoracic needle biopsy of the lung. J Thorac Dis. 2015; 7(Suppl 4): S304-S316, doi: 10.3978/j.issn.2072-1439.2015.12.16, indexed in Pubmed: 26807279 .

12. Tomiyama N, Yasuhara Y, Nakajima Y, et al. CT-guided needle biopsy of lung lesions: a survey of severe complication based on 9783 biopsies in Japan. Eur J Radiol. 2006; 59(1): 60-64, doi: 10.1016/j.ejrad.2006.02.001, indexed in Pubmed: 16530369.

13. Asano F, Aoe M, Ohsaki Y, et al. Deaths and complications associated with respiratory endoscopy: a survey by the Japan Society for Respiratory Endoscopy in 2010. Respirology. 2012; 17(3): 478-485, doi: 10.1111/j.1440-1843.2011.02123.x, indexed in Pubmed: 22222022.

14. Izumo T, Sasada S, Chavez C, et al. The diagnostic utility of endobronchial ultrasonography with a guide sheath and tomo- synthesis images for ground glass opacity pulmonary lesions. J Thorac Dis. 2013; 5(6): 745-750, doi: 10.3978/j.issn.2072 1439.2013.11.30, indexed in Pubmed: 24409350.

15. Izumo T, Sasada S, Chavez C, et al. The diagnostic value of histology and cytology samples during endobronchial ultrasound with a guide sheath. Jpn J Clin Oncol. 2015; 45(4): 362366, doi: 10.1093/jjco/hyv004, indexed in Pubmed: 25628350.

16. Hayama M, Izumo T, Matsumoto Y, et al. Complications with endobronchial ultrasound with a guide sheath for the diagnosis of peripheral pulmonary lesions. Respiration. 2015; 90(2): 129-135, doi: 10.1159/000431383, indexed in Pubmed: 26112297

17. Ali MS, Trick W, Mba BI, et al. Radial endobronchial ultrasound for the diagnosis of peripheral pulmonary lesions: A systematic review and meta-analysis. Respirology. 2017; 22(3): 443453, doi: 10.1111/resp.12980, indexed in Pubmed: 28177181.

18. Park JS, Kim HK, Choi YS, et al. Unplanned conversion to thoracotomy during video-assisted thoracic surgery lobectomy does not compromise the surgical outcome. World J Surg. 2011; 35(3): 590-595, doi: 10.1007/s00268-010-0913-6, indexed in Pubmed: 21181470.

19. Li S, Lv W, Zhou K, et al. Does the fissureless technique decrease the incidence of prolonged air leak after pulmonary lobectomy? Interact Cardiovasc Thorac Surg. 2017; 25(1): 122-124, doi: 10.1093/icvts/ivx061, indexed in Pubmed: 28379438.

20. Kouritas VK, Kefaloyannis E, Tcherveniakov P, et al. Do pleural adhesions influence the outcome of patients undergoing major lung resection? Interact Cardiovasc Thorac Surg. 2017; 25(4): 613619, doi: 10.1093/icvts/ivx173, indexed in Pubmed: 28962506.

21. Berfield KS, Wood DE. Sublobar resection for stage IA non-small cell lung cancer. J Thorac Dis. 2017; 9(Suppl 3): S208-S210, doi: 10.21037/jtd.2017.03.135, indexed in Pubmed: 28446986.

22. Kuriyama K, Tateishi R, Doi O, et al. Prevalence of air bronchograms in small peripheral carcinomas of the lung on thinsection CT: comparison with benign tumors. AJR Am J Roentgenol. 1991; 156(5): 921-924, doi: 10.2214/ajr.156.5.2017952, indexed in Pubmed: 2017952.

23. Ost D, Fein AM, Feinsilver SH. Clinical practice. The solitary pulmonary nodule. N Engl J Med. 2003; 348(25): 2535-2542, doi: 10.1056/NEJMcp012290, indexed in Pubmed: 12815140 . 